\title{
Development of Electromagnetic Calorimeter Using LYSO Crystals for the COMET Experiment at J-PARC
}

\author{
Kou Oishi*广 \\ Kyushu University, Fukuoka, Japan \\ E-mail: koulepp.phys.kyushu-u.ac.jp
}

\begin{abstract}
An electromagnetic calorimeter (ECAL) has been developed for the COMET experiment at JPARC in Japan, which searches for muon-to-electron conversion of a charged lepton flavor violating process with a single event sensitivity of $10^{-17}$. The observation of this process forbidden in the standard model (SM) gives a clear evidence of new physics beyond the SM. The experiment aims at achieving a single event sensitivity of $10^{-17}$. The detector system consists of an upstream tracker and the ECAL. The ECAL is required to have an energy resolution $<5 \%$, position resolution $<1 \mathrm{~cm}$, and time resolution $<1 \mathrm{nsec}$ at $105 \mathrm{MeV}$, the signal electrons energy. In order to satisfy those requirements, the ECAL consists of LYSO scintillating crystals. Several ECAL prototypes have been developed and evaluated. They have shown an excellent energy resolution of $4.2 \%$, and position resolution of $7.6 \mathrm{~mm}$ at $105 \mathrm{MeV} / \mathrm{c}$, and time resolution $<1 \mathrm{nsec}$, linearity within $0.5 \%$, and particle identification efficiency $>90 \%$. In this paper the detailed design and performance of the ECAL are described.
\end{abstract}

EPS-HEP 2017, European Physical Society conference on High Energy Physics

5-12 July 2017

Venice, Italy

\footnotetext{
* Speaker.

${ }^{\dagger}$ on the half of the COMET collaboration StrECAL group.
} 


\section{Introduction}

The COMET experiment will search for muon-to-electron conversion process in aluminum nuclei $\left(\mu^{-} \mathrm{Al} \rightarrow \mathrm{e}^{-} \mathrm{Al}\right.$ ) at J-PARC, Japan. Its branching ratio (BR) to muon capture is strongly suppressed to $<10^{-54}$ in the standard model (SM) including neutrino oscillation. Some physics beyond the SM (BSM), such as supersymmetric grand unified theory[1], enhance the BR up to $10^{-15}$, its observation is a clear signal of BSM. The experiment will be performed in two-stages, Phase-I and II (Figure 1), which aim at single event sensitivities of $10^{-15}$ and $10^{-17}$, respectively. To understand systematics from the beam line a beam profile measurement will be performed in the Phase- $\mathrm{I}^{1}$. The detector for the beam measurement and the Phase-II consists of a straw tube tracker and electromagnetic calorimeter, called StrECAL (Figure 2).

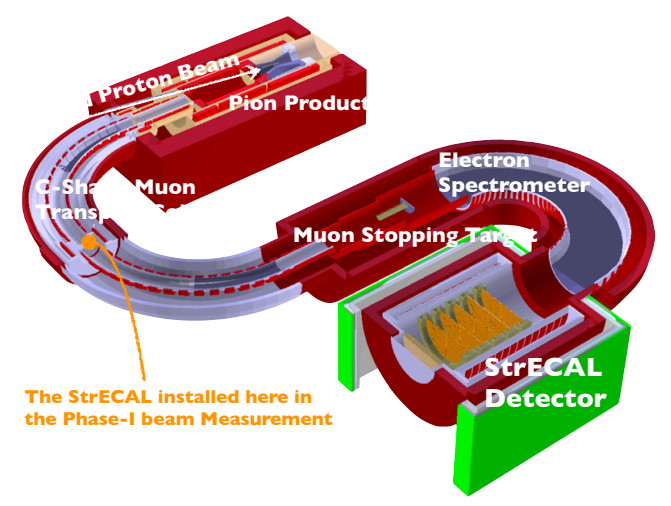

Figure 1: Phase-II beam line

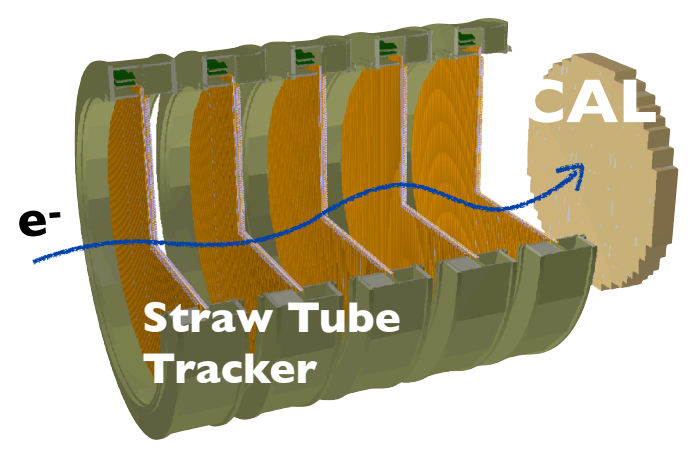

Figure 2: StrECAL detector system

\section{Electromagnetic Calorimeter}

The electromagnetic calorimeter (ECAL) supplies triggers. The trigger rate is dominated by muon decay in orbit $(\mu \mathrm{N} \rightarrow \mathrm{e} v \bar{v} \mathrm{~N})$, electron energy of which reaches close to the signal, $105 \mathrm{MeV}$. Due to the intense beam many event pileups are expected, therefore signal separation and track matching with the tracker are important. The requirement to the ECAL is energy resolution is < $5 \%$, time resolution $<1 \mathrm{nsec}$, and position resolution $<10 \mathrm{~mm}$ at $105 \mathrm{MeV}$. In addition, for the beam measurement the ECAL must distinguish particle kinds.

The COMET ECAL is a segmented array with a diameter of approximately $1 \mathrm{~m}$ consisting of LYSO $\left(\mathrm{Lu}_{2-x} \mathrm{Y}_{x} \mathrm{SiO}_{5}\right)$ inorganic scintillating crystals with dimensions of $2 \times 2 \times 12 \mathrm{~cm}^{3}$. They have a high light yield and short decay constant compared to conventional inorganic scintillators. Since the StrECAL is operated in a vacuum of $<100 \mathrm{~Pa}$ and magnetic field of $1 \mathrm{~T}$, Avalanche Photo Diode (APD) of Hamamatsu Photonics with a sensitive area of $10 \times 10 \mathrm{~mm}^{2}$ is adopted as the photodetector. The array is divided into $2 \times 2$ crystals modules, Figure 3 illustrates the design of them. A crystal is wrapped by two layers of PTFE and an ESR sheet of 3M. A PCB is attached on the back, on which an APD and slow control modules, LED and temperature sensor for gain monitoring, are mounted. $2 \times 2$ crystals are grouped by an aluminized Mylar bag that tightly shrinks

\footnotetext{
${ }^{1}$ In the Phase-I the beam line will be built until a half of the transport solenoid. A cylindrical drift chamber is used for the physics measurement.
} 
by heating. Cables of the slow control modules are clustered by an intermediate $\mathrm{PCB}$, and taken out from the vacuum region together with signal cables from the APDs via a feedthrough PCB. A preamplifier and PCB mediating high voltage and slow control lines are directly attached there to minimize electrical noise. The latest ECAL prototype constructed in 2017 are shown in Figure 4.

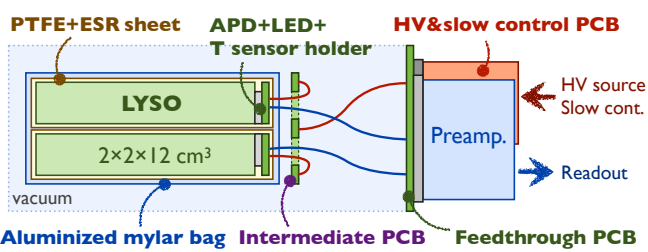

Figure 3: A $2 \times 2$ module

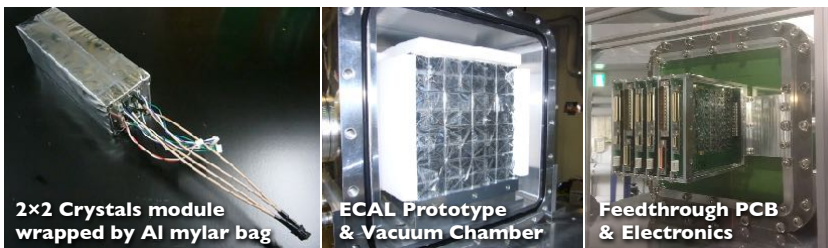

Figure 4: Pictures of the ECAL prototype

We have been originally developing electronics specialized for the ECAL. APD signals are processed by a fast low-noise preamplifier and readout by a waveform digitizing board containing the domino ring sampler (DRS4) chips developed by Paul Scherrer Institut (PSI), Switzerland. that possesses daisy-chain function for gigabit ethernet data transfer. Sequentially connected ones can be readout by single TCP/IP port. Trigger and slow control electronics are also being developed.

\section{ECAL performance evaluation}

In order to evaluate the resolution and particle identification (PID) efficiency, we carried out experiments by using electron beams at Tohoku University, Japan in 2015 and by using $\pi / \mu / \mathrm{e}$ beams at at PSI, Switzerland in 2015, respectively. Figure 5 shows the results: energy resolution of $4.2 \%$ and position resolution of $7.6 \mathrm{~mm}$ at $105 \mathrm{MeV} / \mathrm{c}$, and PID efficiency $>90 \%$ against to all of $\pi / \mu / \mathrm{e}$ over entire the momentum range. The PID efficiency was evaluated by including time-of-flight information in the StrECAL from a full Monte Carlo simulation based on the Geant4 toolkit. In addition, the time resolution and linearity were estimated at $<1 \mathrm{nsec}$ and within $0.5 \%$, respectively. As a conclusion, the basic performance satisfies the requirements.

The latest ECAL prototype was developed and tested in March 2017 together with a straw tracker prototype. This was the first performance evaluation experiment for a combined system of StrECAL prototypes. The data analysis is currently ongoing.
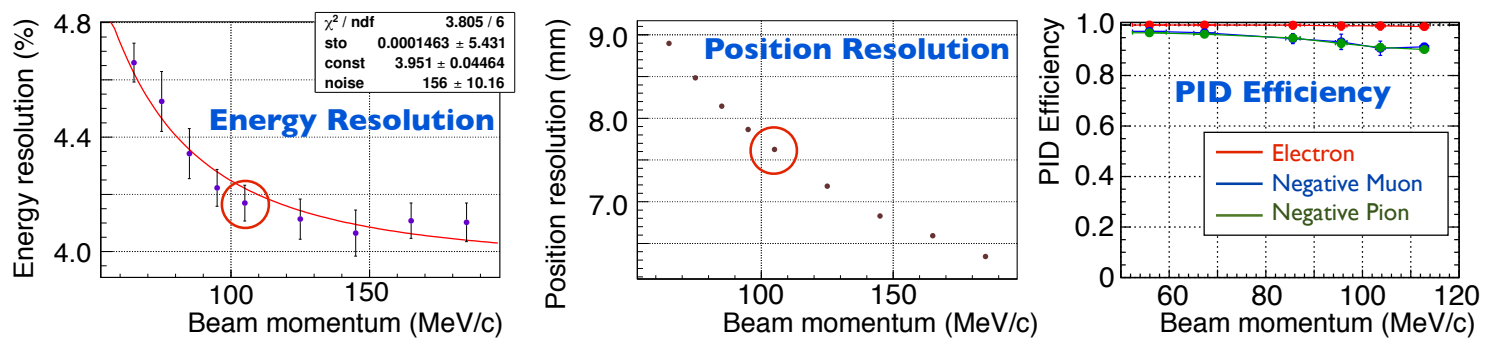

Figure 5: Energy and position resolutions (2016), and PID efficiency (2015)

\section{References}

[1] Y. Kuno and Y.Okada, Rev. Mod. Phys. 73, pp.151-202 (2001) 